needed before this aspect can be resolved. It seems that we may now be in sight of a possible resolution of the apparent discrepancy inherent in the observation that applied gibberellin will give a massive growth stimulation in tissues containing quantities of extractable activity sufficient to sustain the maximum potential growth rate. There could also be an acceptable explanation for the interchangeability of red light and exogenous gibberellin in the control of leaf unrolling in dark-grown cereals (Loveys and Wareing, Planta (Berl.), 98, 117; 1971).

Compartmentalisation of endogenous plant growth regulators coupled with environmentally-mediated release mechanisms offers an attractive solution to these, and many other, problems of plant development. The prospects are exciting and there are indications that the existence of such a system for leaf gibberellins may soon be unequivocally established.

\section{Polyamines and growth control}

\section{from Robert Shields}

Although it has been known for some time that polyamine levels alter with changing rates of cell proliferation, the precise role (if any) that these molecules have in cell regulation is still a mystery.

The first step in the synthesis of polyamines is the decarboxylation of ornithine to form putrescine by ornithine decarboxylase (ODC) which is the rate limiting enzyme for polyamine synthesis. This enzyme is somewhat unusual in that its activity in a given tissue can vary by more than 20 -fold in different conditions and it must also hold the world record for the fastest vanishing mammalian enzyme activity with a half life that can be as short as five minutes in some conditions. It is then hardly surprising that ODC has attracted so much attention. The second enzyme of the pathway, Sadenosyl methionine decarboxylase (SAM decarboxylase) takes part in the conversion of putrescine to spermidine and the sequence is completed with the conversion of spermidine to spermine. Increased levels of ODC and SAM decarboxylase invariably accompany increased cell proliferation and indeed the levels of these enzymes may be very closely correlated with the specific growth rate of certain cells (Heby et al., J. Cell Physiol., 86, 511; 1975). Presumably it is not the levels of these enzymes themselves that may regulate growth but rather the polyamines they produce. Careful analysis of polyamine concentrations and growth rate show that levels of spermidine seem to be closely linked with cell proliferation rates whereas putrescine and spermine show little or no correlation (Heby et $a l ., o p$. cit). No amount of correlative evidence can prove a regulatory role for polyamines however; clearly a more direct approach is necessary.

If polyamines are growth regulatory then interfering with their synthesis by use of enzyme inhibitors should interfere with cell growth. And this is what happens; cell division proceeds more slowly and in some conditions may cease altogether. Two types of inhibitors have been used; one is the anti-leukaemic agent MGBG which inhibits SAM decarboxylase and the others are various ornithine analogues which inhibit ODC. Using MGBG Fillingame and Morris were able to block the increases in spermidine and spermine that are normally observed after stimulation of lymphocytes by concanavalin A (con A). The increased synthesis and processing of RNA and synthesis of protein brought about by con A continued unimpeded however (Biochemistry, 12, 4479; 1973).

This important observation shows that increases in spermidine and spermine are not necessary for increases in protein and RNA synthesis although a requirement for certain basal levels of polvamines in macromolecular synthesis cannot be ruled out. Although MGBG had no effect on the increased synthesis of RNA and protein the drug could partially inhibit DNA synthesis. The inhibition was somewhat unusual in that the number of lymphocytes that entered DNA synthesis after con A stimulation was unaltered but progress through $\mathrm{S}$ phase to mitosis was impaired (Fillingame et al., Proc. natn. Acad. Sci. U.S.A., 72, 4042; 1975). These results suggested that increased levels of spermine or spermidine might be necessary for normal progress from DNA synthesis to cell division. One possible objection to these experiments is that blocking SAM decarboxylase with MGBG leads to elevation of putrescine levels which could fulfil the functions of spermidine and spermine sufficiently well for increased RNA and protein synthesis but not for DNA synthesis to occur. If this were the case it might be expected that blocking putrescine formation and hence the synthesis of spermidine and spermine might have more profound effects than those obtained with MGBG.

Precisely this line of approach is taken in recently reported experiments using $\alpha$-methyl ornithine to inhibit ODC and hence polyamine synthesis (Mamont et al., Proc. natn. Acad. Sci. U.S.A., 173, 1626; 1976). These workers used a suspension culture of a hepa- toma cell line (HTC) whose growth rate is slowed at high cell densities; diluting the culture leads to a rise in ODC and polyamine levels followed by increased rates of DNA synthesis and cell division. If the culture is diluted in the presence of $\alpha$-methyl ornithine, the cells enter DNA synthesis and divide normally but subsequent rounds of DNA synthesis and cell division are inhibited and cell proliferation ceases. Examination of polyamine levels after culture dilution shows that putrescine concentrations follow the activity of ODC (McCann et al., Biochem, biophys. Res. Commun., 64, 336; 1975), peaking some time before the increase in DNA synthesis. In the presence of $\alpha$-methyl ornithine putrescine concentrations fall very rapidly but no inhibition of DNA synthesis occurs until the spermidine levels have dropped significantly by which time the cells would normally have entered the second wave of cell division. The failure of the cells to enter this second round of cell division can be directly related to low spermidine levels as spermine concentrations remain relatively unchanged. Taken together with the results from lymphocytes and the close correlation between spermidine levels and growth rate it seems that of the polyamines only spermidine may play a part in DNA synthesis and cell division. A certain basal level of polyamines may be necessary for normal growth, however, and experiments using inhibitors of polyamine synthesis and the selection of cell mutants with altered polyamine metabolism may shed some light on the normal functions of these curious compounds.

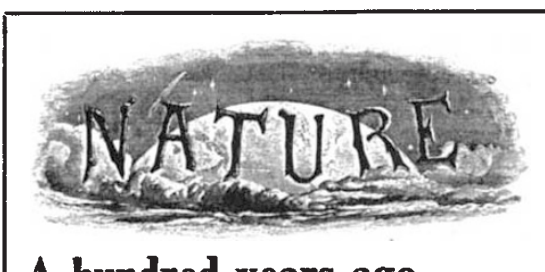

\section{A hundred years ago}

THE comparatively infrequent employment of electric light, considering the great success achieved in its production, would at first sight appear to be due to something in the application of the electricity itself. It has been repeatedly and satisfactorily proved that a continuous and powerful light can be produced by electricity, and the question naturally arises, Why is it not more frequently employed for practical purposes?

Unquestionably the first experiments with electric light were not successful, but this is generally the case with new inventions. Unfortunately, however, a feeling seems to have arisen directly against the application of electricity for lighting purposes, or at any rate against the employment of the existing apparatus in the hope that more perfect may soon be invented.

from Nature, 14, 133-144, June 8, 1876 